\title{
Estradiol induces HOTAIR levels via GPER- mediated miR-148a inhibition in breast cancer
}

\author{
Sifeng Tao*, Haifei He and Qiang Chen
}

\begin{abstract}
HOTAIR plays an important role in the regulation of cancer cell proliferation and cancer invasion in breast cancer. The up-regulation of HOTAIR has been reported in both estrogen receptor (ER) positive and triple-negative (TN) breast cancer. It has been reported that HOTAIR is regulated by estrogen (E2) via ERs in ER-positive breast cancer. However, it is unknown how HOTAIR is regulated in TN breast cancer. In this study, we found that HOTAIR was increased in the peripheral blood mononuclear cells and cancer tissues from breast cancer patients, and was especially higher in patients with metastatic breast cancer. In addition, we found that estrogen promoted HOTAIR through its receptor GPER and estrogen-induced breast cancer cell migration was reversed by deleting HOTAIR in TN breast cancer cells MDA-MB-231and BT549. Furthermore, we identified that E2-GPER induces the level of HOTAIR through the suppression of miR-148a. miR-148a level was negatively correlated with HOTAIR level in breast cancer patients. After the mutation of the predicted miR-148a binding sites in HOTAIR, miR-148a had no effect on HOTAIR. In conclusion, our findings offer important new insights into the ability of estrogenic GPER signaling to increase the HOTAIR level by inhibiting miR-148a in breast cancer.
\end{abstract}

Keywords: HOTAIR, Estrogen, miR-148a, Breast cancer

\section{Introduction}

Breast cancer is one of the most common malignant diseases in women. However, the molecular pathogenesis of breast cancer remains poorly defined due to its heterogeneity [1]. Despite advances in the treatment of breast cancer, the effective control of metastasis remains a complex problem. It has been reported that over $90 \%$ of the deaths of cancer patients are caused by metastasis, which is formed by the spread of disseminated primary tumor cells to distant anatomic sites [2]. Finding new modalities to treat patients who do not respond to conventional treatments has become increasingly important.

Non-coding RNA has become the focus of "next generation" biology. Non-coding RNA includes microRNAs (miRNAs) and long non-coding RNAs (lncRNAs). Roles for miRNAs have been demonstrated in the regulation of a broad range of biological activities and diseases [3,4]. More recently, thousands of lncRNAs, which are transcribed non-coding RNAs that have more than 200 nucleotides, were discovered and implicated in a

\footnotetext{
*Correspondence: SifengTao1023@163.com

Department of Surgical Oncology, Second Affiliated Hospital, School of

Medicine, Zhejiang University, 88 Jiefang Road, Hangzhou 310009, China
}

variety of biological processes [5,6]. In these thousands of lncRNAs, HOTAIR is a star that is highly expressed in primary breast tumors [7], hepatocellular carcinoma [8], colorectal cancer [9] and gastrointestinal stromal tumors [10]. HOTAIR expression is augmented in primary breast tumors and metastases, and HOTAIR expression level in primary tumors is a powerful predictor of metastases and death $[7,11]$.Therefore, HOTAIR may be a potential therapy target in breast cancer. HOTAIR promotes cancer progression in various ways, including dependents EZH2 to promote cell cycle progression [12], regulating PTEN methylation [13] and maintaining the stemness of cancer cells [14]. However, the mechanism by which HOTAIR increases in breast cancer is unknown.

The hormone estrogen (17 $\beta$-estradiol, E2) has a key role in cell prolife[ration and differentiation through receptor binding and activation [15-17]. The effects of E2 have been widely analyzed in the human mammary gland, where it is responsible for normal epithelial growth and for the development of $70-80 \%$ of human breast cancer tumors [18]. Approximately $70 \%$ of human breast cancer is estrogen receptor- $\alpha$ positive $(E R+)$ and 
up to $20 \%$ of breast cancer is triple-negative breast cancer (TNBC) [19].

In ER-positive breast cancer, HOTAIR is transcriptionally induced by E2 through multiple functional estrogen response elements (EREs) in the promoter region [20]. However, as a highly aggressive breast cancer subtype, TNBC lacks a known signaling pathway amenable to targeted therapy. G-protein-coupled estrogen receptor1 (GPER, formerly known as GPR30) has attracted increasing interest, considering its ability to mediate estrogenic signaling in breast cancer [21]. GPER has also been proposed as a candidate biomarker in triplenegative breast cancer [22]. In addition, in our previous study, we found that E2 can regulate miR-148a expression through GPER [23]. Since HOTAIR increases in both ER-positive and TN breast cancer [24,25], we supposed that estrogen may regulate HOTAIR expression through GPER.

To study whether HOTAIR is regulated by E2 via GPER in breast cancer cells, we measured the mRNA levels of HOTAIR in breast cancer cells after treatment with E2. Furthermore, we investigated the regulation mechanism of E2 on HOTAIR expression. We found that E2 up-regulated HOTAIR in breast cancer cells through GPER via the suppression of miR-148a. Taken together, we are reporting a new mechanism of E2 regulating HOTAIR expression in breast cancer.

\section{Materials and methods}

\section{Patients and sample collection}

Tumor and blood samples were obtained from breast adenocarcinoma patients before surgical or other treatment at Zhejiang University Medical School's Affiliated Second Hospital. Tissue and blood samples were derived from two entirely independent populations. Each patient gave written informed consent. The migration status of tumor was determined by sentinel lymph node biopsy. This study was approved by the Institutional Review Board. The clinicopathologic data are stored in a database in accordance with hospital privacy rules and are summarized in Table 1. All tissue samples were stored in liquid nitrogen within 15 minutes after excision (median delay of 9 minutes). Healthy control tissue was obtained from breast reduction surgery. None of the control samples showed pathological changes. In total, 20 tumor samples and 20 healthy control samples were included.

\section{PBMC isolation}

The blood was collected from a cubital vein with an anticoagulant (heparin sodium) and processed immediately. The controls and patients were matched for age and gender where possible. Peripheral blood mononuclear

\section{Table 1 Clinical variables}

\begin{tabular}{ll}
\hline Breast cancer variables & \\
$\quad$ Age (years) median (range) & 52.43 \\
Tumor size & 8 \\
$<2 \mathrm{~cm}$ & 12 \\
$>2 \mathrm{~cm}$ & \\
Metastasis status & 10 \\
$\quad$ Metastasizing tumors & 10 \\
Non-metastasizing tumors & \\
Estrogen receptor status & 6 \\
$\quad$ Positive & 14 \\
$\quad$ Negative & \\
Tumor type & 20 \\
Invasive ductal carcinomas & 124
\end{tabular}

cells (PBMCs) were separated by centrifugation on Ficoll gradient.

\section{Cell cultures}

MDA-MB-231and BT549 cells were obtained from the Cell Bank of the Chinese Academy of Sciences (Shanghai, China). All cells were maintained in a humidified incubator at $37^{\circ} \mathrm{C}$ and $5 \% \mathrm{CO}_{2}$. For the E2 (Sigma-Aldrich, USA) stimulation experiments, the cells were cultured for at least 3 days in phenol red-free RPMI1640 with 5\% dextrancoated charcoal-treated serum before the E2 treatment.

\section{RT-PCR and real-time PCR}

RNA was extracted using TRIzol. Total RNA $(1 \mu \mathrm{g})$ was reverse-transcribed using a RevertAid First Strand cDNA Synthesis Kit (Fermentas). HOTAIR and miR-148a were measured using qRT-PCR (Roche). The expression of HOTAIR was determined in triplicate in three to six separate experiments and normalized using GAPDH, and miR-148a was normalized using U6. Real-time PCR was performed in the ABI PRISM 7300 Sequence Detection System 2.1 (PE Applied Biosystems) using relative quantification. Analysis and fold differences were determined using the comparative cycle threshold (CT) method. Fold change was calculated from the $\Delta \Delta C T$ values with the formula $2^{-\triangle \triangle} \mathrm{CT}$.

The primers are miR-148a -F:5'-ACACTCCAGCTGG GACAAAGTTCTG-3'; miR-148a -R:5' - CTCAACTGGT GTCGTGGAGTCGGCAATTCAGTTGAGTCAGTGCAC -3'; U6 -F:5' -CTCGCTTCGGCAGCACA-3'; U6- R:5'-AA CGCTTCACGAATTTGCGT-3'; HOTAIR -F:5' -TTTG GACTGTAAAATATGGC-3'; HOTAIR -R:5'-TTCTGA CACTGAACGGACT-3'; GAPDH-F:5' - GTGAAGCAGGC GTCGGA -3' GAPDH-R:5' - AGCCCCAGCGTCAAAGG -3'. 


\section{RNA oligonucleotides and transfection}

The siRNA sequences targeting human HOTAIR (siHOTAIR-1 UAACAAGACCAGAGAGCUGUU; siHOTAIR2 CCACAUGAACGCCCAGAGAUU; siHOTAIR 3 GA ACGGGAGUACAGAGAGAUU) or negative control RNA (NC CUACAACAGCCACAACGUCdTdT) were designed and produced by Genepharma (Shanghai, China). siRNA transfection was performed using Lipo2000 (QIAGEN). siRNAs with $20 \mathrm{nmol} / \mathrm{L}$ were used for transfection in a serum-free medium. The total RNA was prepared $24 \sim 48$ hours after transfection and used for quantity RT-PCR analysis.

\section{Migration and invasion assays}

For the transwell migration assay, the breast cancer cells were trypsinized and placed in the upper chamber of each insert (Corning, Cambridge, USA) containing the non-coated membrane. Then, a medium supplemented with $20 \%$ fetal bovine serum $(600 \mu \mathrm{l})$ was added to the lower chambers. After 24, 36 and 48 hours of incubation at $37^{\circ} \mathrm{C}$, the upper surface of the membrane was wiped with a cotton tip, and the cells attached to the lower surface were stained for 10 min with crystal violet. The cells in five random fields of view at $\times 100$ magnification were counted and expressed as the average number of cells per field of view. All assays were performed in triplicate.

\section{Immunoblotting}

MDA-MB-231 cells were stimulated with $1 \mu \mathrm{M}$ G1 (Sigma-Aldrich, USA) with or without 100 nM G15 (Sigma-Aldrich, USA) for $6 \mathrm{~h}$. Then cell lysates were harvested in a cell lysis buffer (Boster, Wuhan, China), dissolved in 9\% SDS-PAGE buffer, and subjected to western blotting using primary detection antibodies against total or phosphorylated ERK1/2 (diluted 1:1000; BioWorld, St Louis Park, MN, USA). Membranes were incubated overnight at $4^{\circ} \mathrm{C}$ before incubation with the appropriate HRPconjugated secondary antibodies. Immunodetection was conducted using the enhanced chemiluminescence system (Amersham Pharmacia Biotech).

\section{Luciferase reporter assay}

The full length of HOTAIR was amplified and cloned into downstream of PGL3-control vector (Promega). Cells plated on 24-well plates were transfected with $100 \mathrm{ng}$ plasmid and $200 \mathrm{nmol} / \mathrm{L}$ of miR-148a mimics (RiboBio Co., Ltd., Guangzhou, China), miR-148a inhibitors (RiboBio Co., Ltd., Guangzhou, China) or their negative control (RiboBio Co., Ltd., Guangzhou, China). The miRNA mimics were a sequence of synthetic mature miRNAs used for functional studies of miRNAs. The miRNA inhibitors were synthetic antisense oligonucleotides, which are complementary to the mature
miRNA sequence and used for loss-of-function studies of miRNAs. After 48 hours, the cells were lysed and assayed with a dual luciferase assay (Promega) according to the manufacturer's instructions. For HOTAIR promoter analysis, the HOTAIR promoter ( -35 to -2286$)$ was amplified and cloned into a PGL3-basic vector (Promega).Transfection efficiency was estimated by cotransfecting the cells with SV-40 Renilla luciferase. Luciferase activity was measured using the dual luciferase assay system (Promega) and a 96-well luminometer (Fluoroskan Ascent Fl, Labsystems). Three independent experiments were performed in triplicate.

\section{Statistics}

A statistical analysis was performed using Prism 5.0. One-way analysis of variance (ANOVA) and Tukey post hoc tests were used for comparisons within a group. The student $\mathrm{t}$ test was used for comparing two different treatments for one cell. All tests were two-sided and $\mathrm{p}<0.05$ was considered significant. The association analysis was evaluated with Fisher's exact test.

\section{Results}

The HOTAIR level was increased in the PBMCs and tumor tissues from the breast cancer patients

To investigate whether the expression of HOTAIR is changed in breast cancer patients, we collected PBMCs from 20 breast cancer patients and 20 normal women, and measured the HOTAIR levels using real-time PCR. The results showed that the HOTAIR levels in breast cancer were significantly higher than those in normal women ( $p=0.0007$ ) (Figure 1A). Moreover, we measured the expression of HOTAIR in breast cancer tissues $(\mathrm{n}=20)$ and normal breast tissues $(\mathrm{n}=20)$. As shown in Figure 1B, HOTAIR was significantly increased in the breast cancer tissues $(p=0.0003)$. In addition, we also compared the differential expression of HOTAIR in the PBMCs and breast cancer tissues from the patients with migrated breast cancer and non-migrated breast cancer. The results showed that HOTAIR expression was significantly up-regulated in the PBMCs $(\mathrm{p}=0.0285)$ (Figure 1C) and tissues $(\mathrm{p}=0.0048)$ (Figure 1D) from the migrated breast cancer patients.

\section{E2-induced HOTAIR increases the migration of breast cancer cells}

To determine whether HOTAIR is a target gene of E2 in triple-negative (TN) breast cancer cells, TN breast cancer cells MDA-MB-231 and BT549 were treated with E2, and HOTAIR expression was measured by using quantitative PCR. HOTAIR expression was significantly up-regulated by E2 in both cell lines (Figure 2A and B). Dose-response experiments revealed maximal HOTAIR reduction with $100 \mathrm{nM}$ after $24 \mathrm{~h}$ of treatment in the 



Figure 1 HOTAIR level increased in the PBMCs and tumor tissues from the breast cancer patients. A): PBMCs were collected from 20 patients with breast cancer and 20 healthy women. Then the expression of HOTAIR was detected using real-time PCR. B): Fresh 20 human breast cancer tissues and 20 normal human breast tissues were collected and HOTAIR expression was assessed with real-time PCR. C): PBMCs were collected from 10 patients with breast cancer migration and 10 without migration. Then the expression of HOTAIR was detected using real-time PCR. D): Fresh 10 breast cancer tissues from patients with breast cancer migration and 10 without migration were collected and HOTAIR expression was assessed by using real-time PCR. ${ }^{*} p<0.05,{ }^{* *} p<0.01$.

MDA-MB-231 and BT549 cells, about 2.7 fold of the control group (Figure 2A). Because $10 \mathrm{nM}$ is close to physiological concentration, this concentration is used in the following experiment. Then we detected the E2induced migration in the breast cancer cells. As shown in Figure 2C, the migration of the MDA-MB-231 $(\mathrm{p}<0.01)$ and BT549 $(\mathrm{p}<0.01)$ cells was significantly increased after treatment with $10 \mathrm{nM}$ E2 for $36 \mathrm{~h}$. Furthermore, we investigated the roles of HOTAIR in E2-induced cancer cell migration. Before E2 treatment, HOTAIR-specific siRNAs (si-HOTAIR1, si-HOTAIR2 and si-HOTAIR3) were transfected into MDA-MB-231 and BT549 cells. The efficiency of the HOTAIR-specific siRNAs was checked with qRTPCR after transfecting into MDA-MB-231 for $24 \mathrm{~h}$. As shown in Figure 2D, si-HOTAIR1, si-HOTAIR2 and siHOTAIR3 inhibited the level of HOTAIR to $21.2 \%$, 18.9\% and $17.9 \%$, respectively. After deleting HOTAIR with specific siRNAs, the E2-induced cancer cell migration was reversed (Figure 2E).

\section{GPER mediates the promotion effect of E2 on HOTAIR levels}

Because classical estrogen receptors were negative in the TN breast cancer cells, we speculated that GPER may mediate the effects of E2. First, we checked the inhibition effect of G15 (GPER inhibitor) on GPER signaling.
Luo et al. reported that GPER signaling can activate ERK signaling [26]. We treated the MDA-MB-231 cells with GPER agonist G1 $(1 \mu \mathrm{M})$ with or without $100 \mathrm{nM}$ G15 for $6 \mathrm{~h}$. Then we detected the expression of the p-ERK levels. As shown in Figure 3A, the p-ERK level was increased after G1 treatment, while it was reversed by G15. Then, the MDA-MB-231 and BT549 cells were pretreated with $100 \mathrm{nM}$ G15 for 6 h before E2 treatment. As shown in Figure 3B, E2 can induce HOTAIR levels in both MDA-MB-231 and BT549 cells. However, G15 blocked the E2-induced increase of HOTAIR, indicating that GPER mediated this response. Furthermore, we wanted to investigate whether E2/GPER induce HOTAIR by affecting the HOTAIR promoter. First we constructed the HOTAIR promoter including PGL3basic luciferase reporter plasmid and transfected them into the MDA-MB-231 and BT549 cells, then $10 \mathrm{nM} \mathrm{E2}$ was added into these transfected cells. After E2 treatment for $24 \mathrm{~h}$, the luciferase activity was detected. As shown in Figure 3C, E2 had no effect on luciferase activity, indicating that E2/luciferase may induce HOTAIR expression in a different way.

\section{miR-148a targets HOTAIR in breast cancer cells}

It has been reported that lncRNAs can be regulated by miRNAs. We have found that E2 regulated miR-148a 


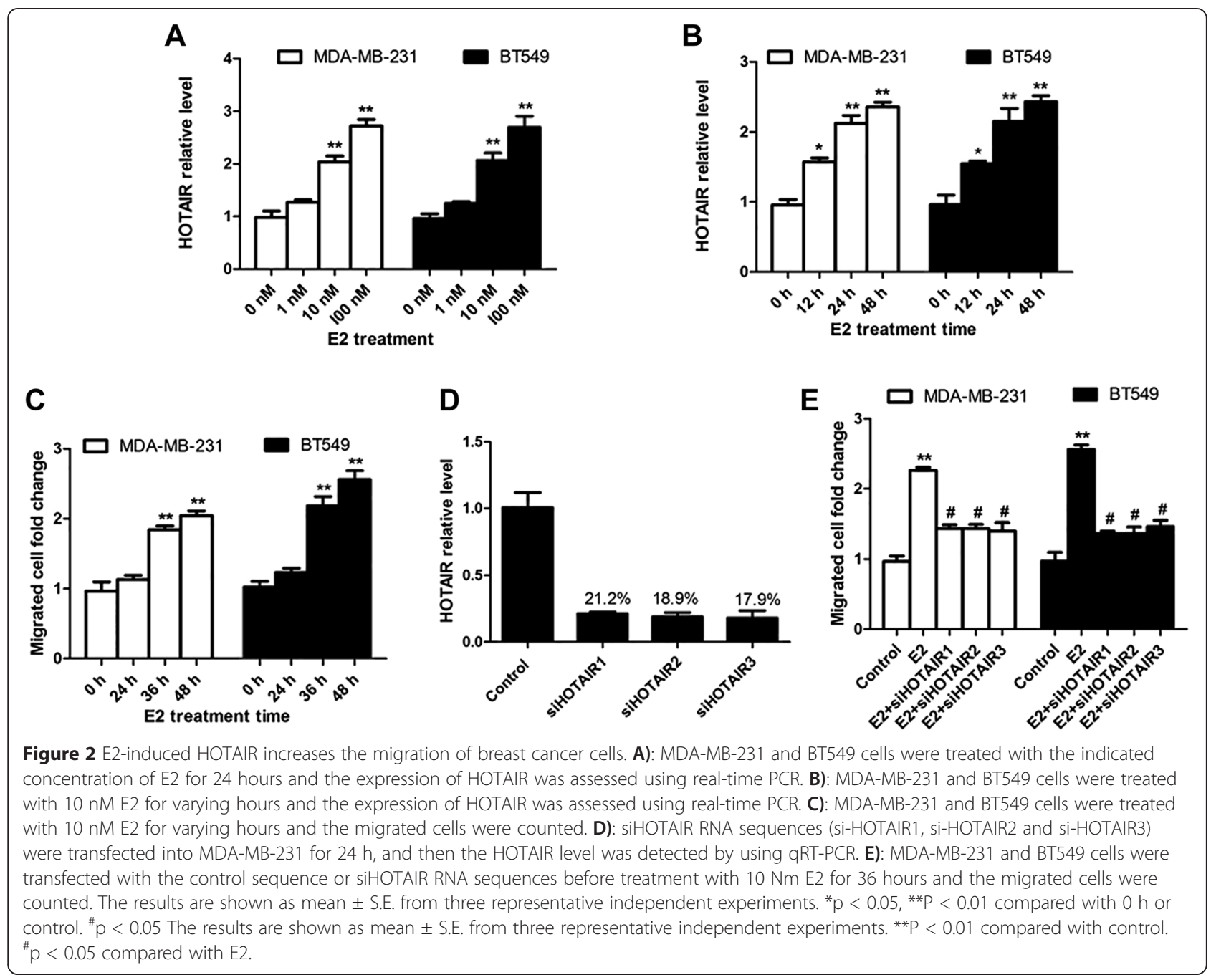

expression in breast cancer cells. miR-148a is an antimigration miRNA in cancer cells. Of note, we predicted that HOTAIR has miR-148a binding sequences using DIANA Tools (http://diana.imis.athena-innovation.gr/ DianaTools/index.php? $r=\operatorname{lncBase} /$ indexbio) (Figure 4A). Furthermore, we found that the miR-148a level in the PBMCs from the breast cancer patients was also negatively correlated with the HOTAIR level $\left(R^{2}=0.6492\right.$, $\mathrm{P}<0.001$ ) (Figure 4B). The miR-148a level in the cancer tissues from the breast cancer patients was also negatively correlated with the HOTAIR level $\left(\mathrm{R}^{2}=\right.$ $0.6251, \mathrm{P}<0.001$ ) (Figure 4C). Next, before the luciferase reporter assay, we checked the transfection efficiency of the miR-148a mimics and miR-148a inhibitors. As shown in Figure 4D, the miR-148a level was 97.3\%, $1697 \%$ and $34.4 \%$ compared to control after transfection with negative miRNA, miR-148a mimics and miR-148a inhibitors, respectively. The luciferase reporter assay demonstrated that miR-148a significantly suppressed the expression of a luciferase reporter gene fused full sequence of HOTAIR, which could be reversed by further introduction of the miR-148a inhibitor in the MDA-MB-231 and BT549 cells (Figure 4E). To further identify that the sequence shown in Figure $4 \mathrm{~A}$ was miR-148a binding sites, we muted TGCAC (11851189) to CCTTG. Then we found that miR-148a could not affect the luciferase activity after the mutated HOTAIR was cloned into the luciferase reporter plasmid (Figure 4F). Furthermore, we found that E2induced HOTAIR could be reversed by adding miR148a (Figure 4G).

\section{Discussion}

LncRNAs are of high interest as potential breast cancer therapeutics. However, their expression and function in breast cancer still need to be elucidated. Estrogen signaling is important in the development and progression of breast cancer [15]. HOTAIR, one of the important 
A

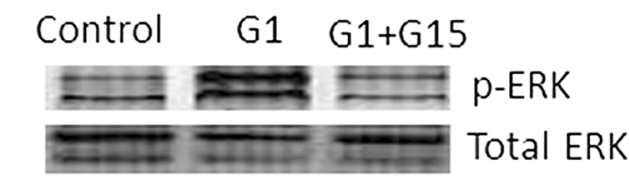

C

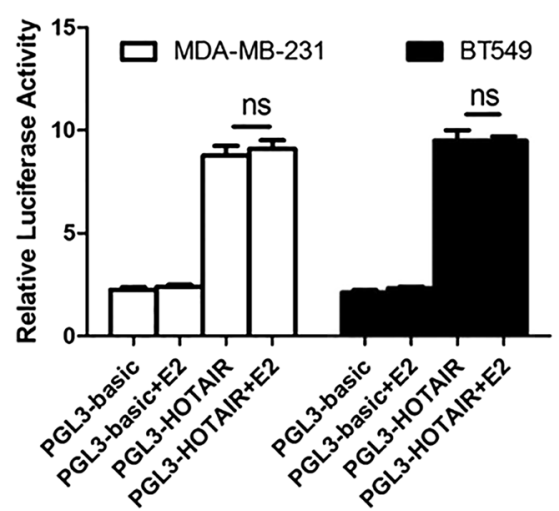

B

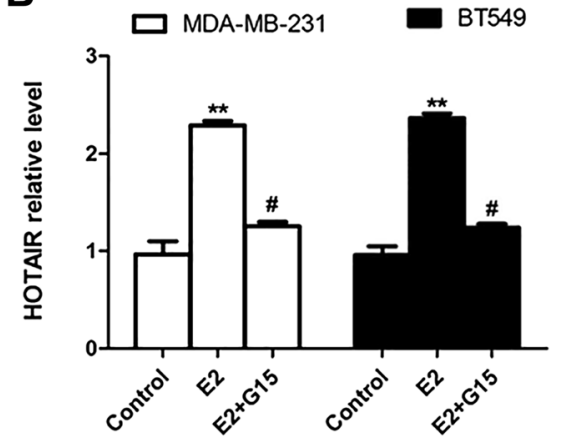

Figure 3 GPER mediates the promotion effect of E2 on HOTAIR expression. A): MDA-MB-231 cells were treated with $1 \mu$ M G1 with or without 100 nM G15 for 6 h. Then the expression of the p-ERK level was checked with a western blot. B): MDA-MB-231 and BT549 cells were pretreated with 100 nM G15 for $6 \mathrm{~h}$ before the addition of $10 \mathrm{nM}$ E2 for $24 \mathrm{~h}$. Then the expression of HOTAIR was determined by real-time PCR. C): The HOTAIR promoter sequence was cloned into PGL3-basic luciferase reporter plasmid, and then the control plasmid and HOTAIR promoter including plasmid were transfected into the MDA-MB-231 and BT549 cells. Then 10 nM E2 was added into these transfected cells. After E2 treatment for 24 h, the luciferase activity was detected. The results are shown as mean \pm S.E. from three representative independent experiments. ${ }^{* *} \mathrm{P}<0.01$ compared with control. ${ }^{*} p<0.05$ compared with E2. ns $p>0.5$.

lncRNAs in the promotion of breast cancer migration, increases in both ER-positive and TN breast cancer $[24,25]$, indicating that estrogen may regulate HOTAIR in a different way other than through ER. In the present study, we found that HOTAIR was increased in breast cancer patients, and was especially higher in migrated breast cancer. In addition, we found that estrogen promoted HOTAIR through its receptor GPER and estrogen-induced breast cancer cell migration was reversed by deleting HOTAIR. Furthermore, we identified that E2-GPER increases the level of HOTAIR through the suppression of miR-148a.

HOTAIR can regulate gene expression through changes in chromatin states and epigenetic modifications [27,28]. Recently, the up-regulation of HOTAIR was observed in several cancers, including breast cancer [7,28-30], hepatocellular carcinoma [11,31], colorectal cancer (CRC) [9,32], pancreatic cancer [33], non-small cell lung cancer (NSCLC) [34] and esophageal squamous cell carcinoma (ESCC) [32,33]. Furthermore, HOTAIR has promoted the migration and invasion of breast carcinoma cells [32], CRC cells [9], pancreatic cancer cells [33], NSCLC cells [34] and
ESCC cells [32,33]. Therefore, to investigate the way HOTAIR regulates cells is very important for cancer clinical therapy.

In ER-positive breast cancer cells, it has been demonstrated that HOTAIR is transcriptionally induced by E2 through multiple functional EREs in its promoter [20]. Estrogen receptors (ERs), along with various ER coregulators such as histone methylases mixed-lineage leukemia 1 (MLL1) and MLL3 and CREB-binding protein/p300, bind to the promoter of HOTAIR in an E2dependent manner. The level of histone $\mathrm{H} 3$ lysine-4 trimethylation, histone acetylation and RNA polymerase II recruitment is enriched at the HOTAIR promoter in the presence of E2. The knockdown of ERs and MLLs downregulated the E2-induced HOTAIR expression [20]. However, in TN breast cancer, HOTAIR is also up-regulated, indicating that HOTAIR may be regulated in a different way. Here, we found that E2 could up-regulate HOTAIR levels through GPER in TN breast cancer cells. Our findings further confirm the important role of GPER in cancer development.

E2 regulates the HOTAIR level through the downregulation of miR-148a. Several miRNAs have been 


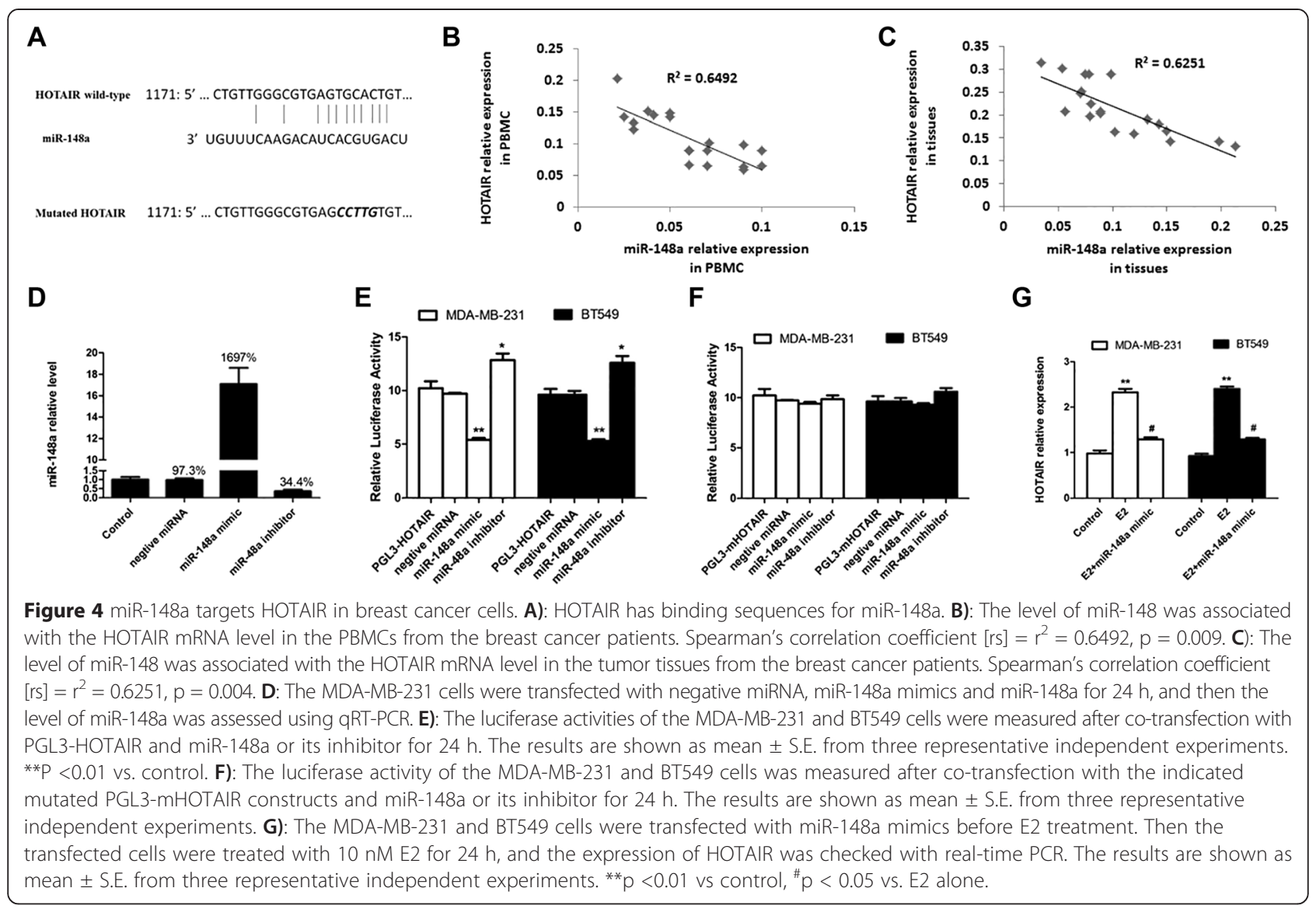

reported to regulate HOTAIR levels. miR-141 suppresses HOTAIR in an Ago2-dependent manner [34]. In addition, HOTAIR can also regulate miRNA levels [35]. miR-148a functions as a tumor suppressor in cancer cells. It has been reported that miR-148a inhibits tumor metastasis by targeting IGF-IR and IRS1 [36]. Moreover, miR-148a suppresses the epithelialmesenchymal transition and metastasis of hepatoma cells by targeting Met/Snail signaling [37]. We found



Figure 5 Model of E2-induced breast cancer cell migration via up-regulation of HOTAIR expression. that the miR-148a level was negatively correlated with the HOTAIR level in breast cancer patients. In addition, we predicted and proved that there are miR148a binding sequences in HOTAIR. Tumor suppressor miRNA inhibits tumor promoting lncRNA, which may be an important regulation method in cancer.

In summary, HOTAIR level is increased in breast cancer patients and associated with cancer migration. GRER mediates E2-induced HOTAIR levels in breast cancer cells, and E2/GPER promote HOTAIR levels through miR-148a. Therefore, as shown in Figure 5, E2-ER can promote HOTAIR by binding ERE in the promoter of HOTAIR in ER-positive breast cancer. While in TN breast cancer, E2-GPER promotes HOTAIR by inhibiting miR-148a, which can identify the sequence in HOTAIR.

\section{Conclusions}

HOTAIR is becoming a potential therapy target in many cancers. However, its transcription regulation method is unknown. Our findings offer important new insights into the ability of estrogenic GPER signaling to increase the HOTAIR level by inhibiting miR-148a. These findings provide new targets for breast cancer therapy. 


\section{Competing interests}

The authors declare that they have no competing interests.

\section{Authors' contributions}

SFT and HFH conceived the idea and were principal investigators responsible for directing and conducting the work, analysis \& interpretation of data, and manuscript writing. SFT and QC participated in the conception, research planning and data analysis. HFH and QC were responsible for clinical samples collection and analysis. QC contributed reagents/materials/analysis tools. All authors contributed to the writing of the manuscript. All authors read and approved the final manuscript.

\section{Acknowledgements}

Authors are grateful to patients for their important contribution to this study. We also thank all the members of this team for their hard work and invaluable assistance.

\section{Received: 24 October 2014 Accepted: 14 April 2015 Published online: 25 April 2015}

\section{References}

1. Vargo-Gogola T, Rosen JM. Modelling breast cancer: one size does not fit all. Nat Rev Cancer. 2007;7:659-72.

2. Fidler IJ. The pathogenesis of cancer metastasis: the 'seed and soil' hypothesis revisited. Nat Rev Cancer. 2003;3:453-8.

3. Espinoza-Lewis RA, Wang DZ. MicroRNAs in heart development. Cur Top Dev Biol. 2012;100:279-317.

4. Zhao G, Zhou X, Chen S, Miao H, Fan H, Wang Z, et al. Differential expression of microRNAs in decidua-derived mesenchymal stem cells from patients with pre-eclampsia. J Biomed Sci. 2014;21:81

5. Ulitsky I, Bartel DP. LincRNAs: genomics, evolution, and mechanisms. Cell. 2013;154:26-46.

6. Morris KV, Mattick JS. The rise of regulatory RNA. Nat Rev Genet. 2014;15:423-37.

7. Gupta RA, Shah N, Wang KC, Kim J, Horlings HM, Wong DJ. Long noncoding RNA HOTAIR reprograms chromatin state to promote cancer metastasis. Nature. 2010;464:1071-6.

8. Geng YJ, Xie SL, Li Q, Ma J, Wang GY. Large intervening non-coding RNA HOTAIR is associated with hepatocellular carcinoma progression. J Int Med Res. 2011;39:2119-28.

9. Kogo R, Shimamura T, Mimori K, Kawahara K, Imoto S, Sudo T. Long noncoding RNA HOTAIR regulates polycomb-dependent chromatin modification and is associated with poor prognosis in colorectal cancers. Cancer Res. 2011;71:6320-6.

10. Niinuma T, Suzuki H, Nojima M, Nosho K, Yamamoto H, Takamaru H. Upregulation of miR-196a and HOTAIR drive malignant character in gastrointestinal stromal tumors. Cancer Res. 2012;72:1126-36.

11. Yang Z, Zhou L, Wu LM, Lai MC, Xie HY, Zhang F, et al. Overexpression of long non-coding RNA HOTAIR predicts tumor recurrence in hepatocellular carcinoma patients following liver transplantation. Ann Surg Oncol. 2011;18:1243-50.

12. Zhang K, Sun $X$, Zhou X, Han L, Chen L, Shi Z, et al. Long non-coding RNA HOTAIR promotes glioblastoma cell cycle progression in an EZH2 dependent manner. Oncotarget. 2014 Nov 4. [Epub ahead of print]

13. Li D, Feng J, Wu T, Wang Y, Sun Y, Ren J, et al. Long intergenic noncoding RNA HOTAIR is overexpressed and regulates PTEN methylation in laryngeal squamous cell carcinoma. Am J Pathol. 2013;182:64-70.

14. Pádua Alves C, Fonseca AS, Muys BR, de Barros E, Lima Bueno R, Bürger MC, et al. Brief report: The lincRNA Hotair is required for epithelial-tomesenchymal transition and stemness maintenance of cancer cell lines. Stem Cells. 2013;31:2827-32.

15. Deng $H$, Zhang XT, Wang ML, Zheng HY, Liu LJ, Wang ZY. ER-a36-mediated rapid estrogen signaling positively regulates ER-positive breast cancer stem/ progenitor cells. PLoS One. 2014;9, e88034.

16. Scaling AL, Prossnitz ER, Hathaway HJ. GPER mediates estrogen-induced signaling and proliferation in human breast epithelial cells and normal and malignant breast. Horm Cancer. 2014;5:146-60.

17. Zhao G, Nie Y, Lv M, He L, Wang T, Hou Y. ERß-mediated estradiol enhances epithelial mesenchymal transition of lung adenocarcinoma through increasing transcription of midkine. Mol Endocrinol. 2012;26:1304-15.
18. Meneses-Morales I, Tecalco-Cruz AC, Barrios-García T, Gómez-Romero V, Trujillo-González I, Reyes-Carmona S, et al. SIP1/NHERF2 enhances estrogen receptor alpha transactivation in breast cancer cells. Nucleic Acids Res. 2014;42:6885-900.

19. Yager JD, Davidson NE. Estrogen carcinogenesis in breast cancer. N Engl J Med. 2006;354:270-82.

20. Bhan A, Hussain I, Ansari Kl, Kasiri S, Bashyal A, Mandal SS. Antisense transcript long noncoding RNA (IncRNA) HOTAIR is transcriptionally induced by estradiol. J Mol Bio. 2013;425:3707-22.

21. Tamaki M, Konno Y, Kobayashi Y, Takeda M, Itoga M, Moritoki Y, et al. Expression and functional roles of G-protein-coupled estrogen receptor (GPER) in human eosinophils. Immunol Lett. 2014;160:72-8.

22. Lappano R, Pisano A, Maggiolini M. GPER function in breast cancer: an overview. Front Endocrinol (Lausanne). 2014;5:66.

23. Tao S, He H, Chen Q, Yue W. GPER mediated estradiol reduces miR-148a to promote HLA-G expression in breast cancer. Biochem Biophys Res Commun. 2014:451:74-8.

24. Chisholm KM, Wan Y, Li R, Montgomery KD, Chang HY, West RB. Detection of long non-coding RNA in archival tissue: correlation with polycomb protein expression in primary and metastatic breast carcinoma. PLoS One. 2012;7, e47998.

25. Sørensen KP, Thomassen M, Tan Q, Bak M, Cold S, Burton M, et al. Long non-coding RNA HOTAIR is an independent prognostic marker of metastasis in estrogen receptor-positive primary breast cancer. Breast Cancer Res Trea. 2013;142:529-36.

26. Luo H, Yang G, Yu T, Luo S, Wu C, Sun Y, et al. GPER-mediated proliferation and estradiol production in breast cancer-associated fibroblasts. Endocr Relat Cancer. 2014;21:355-69.

27. Tsai MC, Manor O, Wan Y, Mosammaparast N, Wang JK, Lan F, et al. Long noncoding RNA as modular scaffold of histone modification complexes. Science. 2010;329:689-93.

28. Lu L, Zhu G, Zhang C, Deng Q, Katsaros D, Mayne ST, et al. Association of large noncoding RNA HOTAIR expression and its downstream intergenic CpG island methylation with survival in breast cancer. Breast Cancer Res Trea. 2012;136:875-83.

29. Sorensen KP, Thomassen M, Tan Q, Bak M, Cold S, Burton M, et al. Long non-coding RNA HOTAIR is an independent prognostic marker of metastasis in estrogen receptor-positive primary breast cancer. Breast Cancer Res Treat. 2013;142:529-36.

30. Kim K, Jutooru I, Chadalapaka G, Johnson G, Frank J, Burghardt R, et al. HOTAIR is a negative prognostic factor and exhibits pro-oncogenic activity in pancreatic cancer. Oncogene. 2013;32:1616-25.

31. Liu XH, Liu ZL, Sun M, Liu J, Wang ZX, De W. The long non-coding RNA HOTAIR indicates a poor prognosis and promotes metastasis in non-small cell lung cancer. BMC Cancer. 2013;13:464.

32. Ge XS, Ma HJ, Zheng XH, Ruan HL, Liao XY, Xue WQ, et al. HOTAIR a prognostic factor in esophageal squamous cell carcinoma inhibits WIF-1 expression and activates Wnt pathway. Cancer Sci. 2013;104:1675-82.

33. Li X, Wu Z, Mei Q, Guo M, Fu X, Han W. Long non-coding RNA HOTAIR a driver of malignancy predicts negative prognosis and exhibits oncogenic activity in oesophageal squamous cell carcinoma. Br J Cancer. 2013;109:2266-78.

34. Chiyomaru T, Fukuhara S, Saini S, Majid S, Deng G, Shahryari V, et al. Long non-coding RNA HOTAIR is targeted and regulated by miR-141 in human cancer cells. J Biol Chem. 2014;289:12550-65.

35. Zhang H, Cai K, Wang J, Wang X, Cheng K, Shi F, et al. MiR-7, inhibited indirectly by LincRNA HOTAIR, directly inhibits SETDB1 and reverses the EMT of breast cancer stem cells by downregulating the STAT3 pathway. 2014;32:2858-68.

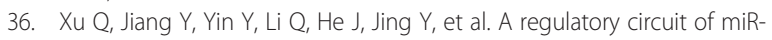
$148 \mathrm{a} / 152$ and DNMT1 in modulating cell transformation and tumor angiogenesis through IGF-IR and IRS1. J Mol Cell Biol. 2013;5:3-13.

37. Zhang JP, Zeng C, Xu L, Gong J, Fang JH, Zhuang SM. MicroRNA-148a suppresses the epithelial-mesenchymal transition and metastasis of hepatoma cells by targeting Met/Snail signaling. Oncogene. 2014;33:4069-76. 